\title{
SHORT COMMUNICATION Relation between sugar-sweetened beverage consumption and micronutrient intake in a prospective study
}

\author{
P Mullie ${ }^{1,2,3}$, E Mertens ${ }^{1,4}$, R Charlier ${ }^{4}$, SS Knaeps ${ }^{4,5}$, J Lefevre ${ }^{4}$ and P Clarys ${ }^{2,3}$
}

\begin{abstract}
Micronutrient dilution following sugar-sweetened beverage (SSB) consumption can lead to a qualitative impoverishment of a dietary pattern. The aim of this prospective study was to investigate the relation between SSB consumption and micronutrients. A total 562 adults were tested in 2002 and 2012 for the same anthropometric, lifestyle and nutritional intake activity parameters. Calcium, iron and magnesium intake decreased with increasing baseline SSB intake, and with increasing SSB consumption during the 10 years. A $100 \mathrm{ml}$ increase in SSB consumption was associated with a $22 \mathrm{mg}$ lower intake of calcium, $0.4 \mathrm{mg}$ of iron and $9 \mathrm{mg}$ of magnesium. There was no relation between vitamins and SSB consumption. In conclusion, there was limited evidence in our study, which suggests SSB have minimal dilutional effect on dietary micronutrient consumption. A major limitation of the present study is that of the original 1569 participants in 2002, 36\% returned for participation in 2012.
\end{abstract}

European Journal of Clinical Nutrition (2018) 72, 170-173; doi:10.1038/ejen.2017.82; published online 24 May 2017

\section{INTRODUCTION}

It has been hypothesized that an increased sugar-sweetened beverage (SSB) consumption in a dietary pattern could create an imbalance in micronutrient intake, because the micronutrient density of SSB is lower than the micronutrient density usually found in natural sugar-containing foods, that is, fruits. This micronutrient dilution, that is, a lower intake of micronutrients for equal energy consumption, could lead to a qualitative impoverishment of a dietary pattern. ${ }^{1-3}$

Three major reviews related added sugar, a major component of SSB, to micronutrient dilution. ${ }^{1-3}$ These reviews concluded that the existing evidence of an inverse relation between reported intakes of added sugar and micronutrients was inconsistent. According to Rennie et al. and Livingstone et al., ${ }^{1-3}$ there were insufficient data and inconsistency between the studies relating added sugars and micronutrient intakes, with no clear evidence of micronutrient dilution. Gibson et al. $^{3}$ concluded that the available evidence did not allow for firm conclusions on an optimal level of added sugar intake for micronutrient adequacy while the inversed trends that exist may have little biological significance.

The aim of the present study was to investigate the influence of time changes in SSB consumption on the intake of micronutrients in a prospective study. We limited our analysis to vitamins and minerals with suboptimal intake in the Belgian population. ${ }^{4}$

\section{MATERIALS AND METHODS}

Data were collected by the Flemish Policy Research Centre Sport, Physical Activity and Health. ${ }^{5}$ One of the aims of this Research Centre was to investigate the relationship between nutritional habits, physical health, mental health and physical fitness among an adult population. The first test moment took place during 2002, the second test moment during
2012. Of the original 1569 participants, 367 men and 195 women returned for participation. The subjects were asked to visit the test laboratory to have anthropometric measurements taken and to complete questionnaires. All the participants signed an informed consent form and received information about the tests and measurements. The study was approved by the ethical and medical committee of the Catholic University of Leuven, Belgium.

The participants completed a 3-day food record. Diet records were analysed using Becel Nutrition software (Unilever Co., Rotterdam, The Netherlands). SSB consumption was defined as the consumption of beverages with added sugar, such as soft drinks; fruit juice and artificially sweetened beverage consumption were excluded. The use of dietary supplements was not assessed.

SPSS 22.0 (SPSS Inc., Chicago, IL, USA) statistics software was used for data analysis. Chi-square tests and paired samples $T$-tests were used for characterization of the participants and to compare body mass index (BMI) between the two test moments. Residual change scores for SSB and total energy intake were created by regressing the follow-up measures onto their respective baseline measures. The residualized change scores can be interpreted as the amount of change between the first and second test moment, independent of baseline levels and are preferable to simple change scores because they eliminate auto-correlated error and regression to the mean effects. The SSB consumption had a non-normal distribution, with a high number of non-consumers. To correct this, the logarithm was used in the multivariate models. Energy intake was expressed in $40 \mathrm{kcal}$ units, which is equal to $10 \mathrm{~g}$ of sugar or $100 \mathrm{ml} \mathrm{SSB}$, allowing interpretation of the regression models. Associations between micronutrients were tested in a multivariate linear regression with the 2012 micronutrient intake as continuous dependent variable, and baseline values for minerals, age, gender, energy intake, baseline log SSB, SSB residuals, energy residuals as independent variables. A two-sided 0.05 level of significance was defined.

\section{RESULTS}

Exposure and outcomes were assessed in 2002 and 2012 in 562 middle-aged adults (367 men and 195 women; Table 1).

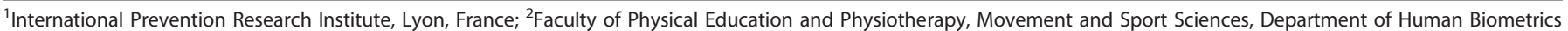

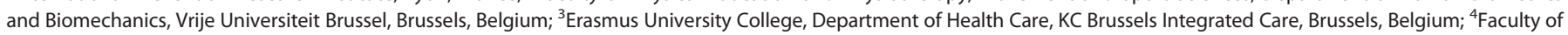

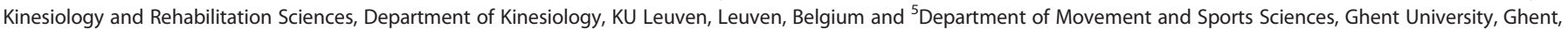

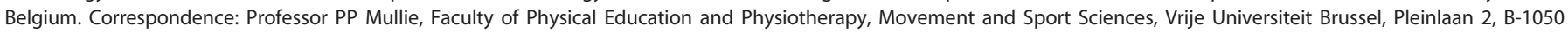
Brussels, Belgium.

E-mail: patrick.mullie@skynet.be

Received 8 October 2016; revised 6 March 2017; accepted 19 April 2017; published online 24 May 2017 
Table 1. Description of the participants to the test periods 2002 and 2012

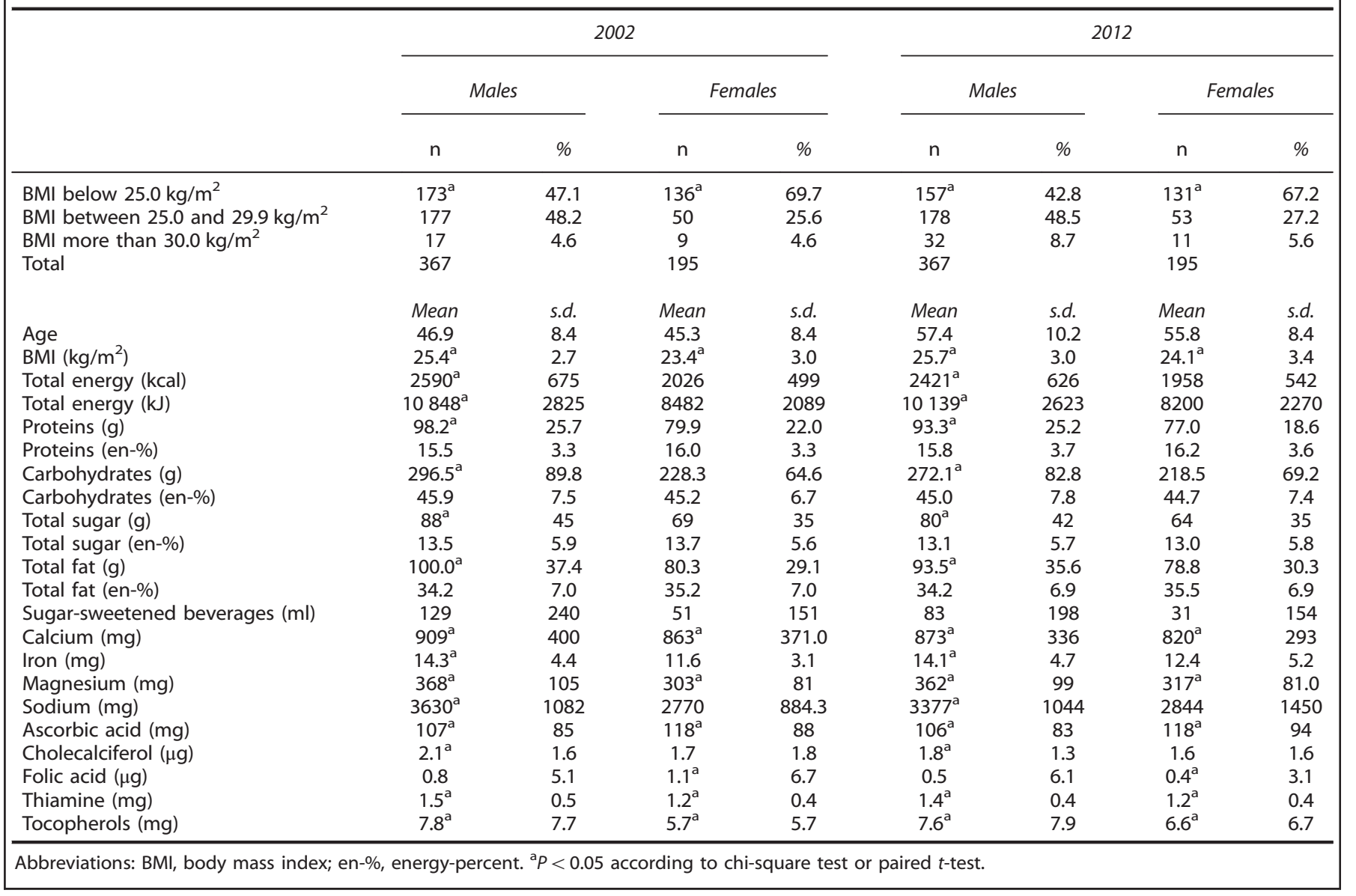

Table 2. Linear regressions with minerals and vitamins at end point as dependent variables, and baseline values for minerals, age, gender, energy intake, log SSB, SSB residuals, energy residuals as independent variables

\begin{tabular}{|c|c|c|c|c|c|c|}
\hline $\begin{array}{l}\text { Dependent } \\
\text { variables }\end{array}$ & $\begin{array}{l}\text { Independent } \\
\text { variables }\end{array}$ & $\begin{array}{l}\text { Linear regression } \\
\text { analysis } \beta \text {-coefficients }\end{array}$ & Standard error & $\begin{array}{l}95 \% \mathrm{Cl} \text {-lower } \\
\text { bound }\end{array}$ & $\begin{array}{l}95 \% \text { Cl-higher } \\
\text { bound }\end{array}$ & P-value \\
\hline Calcium (mg) 2012 & $\begin{array}{l}\text { Constant } \\
\text { Gender (male vs female) } \\
\text { Age } 2002 \\
\text { Calcium (mg) } 2002 \\
\text { Log SSB } 2002 \text { (ml/day) } \\
\text { SSB residuals (ml/day) } \\
\text { Energy } 2002 \text { (unit } 40 \text { kcal) } \\
\text { Energy residuals (unit } 40 \text { kcal) }\end{array}$ & $\begin{array}{l}750.278 \\
7.555 \\
-3.034 \\
0.279 \\
-20.905 \\
-0.220 \\
0.115 \\
9.776\end{array}$ & $\begin{array}{c}71.942 \\
27.530 \\
1.255 \\
0.034 \\
11.222 \\
0.078 \\
0.850 \\
0.865 \\
R^{2}=0.28, F(75\end{array}$ & $\begin{array}{c}608.965 \\
-46.522 \\
-5.499 \\
0.213 \\
-42.947 \\
-0.373 \\
-1.555 \\
8.077 \\
4)=31.33, P<0\end{array}$ & $\begin{array}{cc} & 891.592 \\
61.631 \\
-0.569 \\
0.345 \\
1.137 \\
-0.066 \\
1.785 \\
\\
11.475 \\
001 & \end{array}$ & $\begin{array}{l}<0.001 \\
0.011 \\
0.016 \\
<0.001 \\
0.063 \\
0.005 \\
0.892 \\
<0.001\end{array}$ \\
\hline Iron (mg) 2012 & $\begin{array}{l}\text { Constant } \\
\text { Gender (male vs female) } \\
\text { Age } 2002 \\
\text { Iron (mg) } 2002 \\
\text { Log SSB } 2002 \text { (ml/day) } \\
\text { SSB residuals (ml/day) } \\
\text { Energy } 2002 \text { (unit } 40 \text { kcal) } \\
\text { Energy residuals (unit } 40 \text { kcal) }\end{array}$ & $\begin{array}{c}10.263 \\
0.204 \\
-0.006 \\
0.204 \\
-0.419 \\
-0.004 \\
0.017 \\
0.195\end{array}$ & $\begin{array}{c}1.046 \\
0.405 \\
0.018 \\
0.054 \\
0.164 \\
0.001 \\
0.014 \\
0.013 \\
R^{2}=0.28, F(75\end{array}$ & $\begin{array}{c}8.210 \\
-591.000 \\
-0.042 \\
0.097 \\
-0.742 \\
-0.006 \\
-0.011 \\
0.170 \\
4)=31.33, P<0\end{array}$ & $\begin{array}{c}12.317 \\
0.999 \\
0.030 \\
0.311 \\
-0.097 \\
-0.002 \\
0.044 \\
0.220 \\
001\end{array}$ & $\begin{array}{l}<0.001 \\
0.615 \\
0.752 \\
<0.001 \\
0.011 \\
0.001 \\
0.243 \\
<0.001\end{array}$ \\
\hline Magnesium (mg) 2012 & $\begin{array}{l}\text { Constant } \\
\text { Gender (male vs female) } \\
\text { Age } 2002 \\
\text { Magnesium (mg) } 2002 \\
\text { Log SSB } 2002(\mathrm{ml} / \text { day) }\end{array}$ & $\begin{array}{c}245.198 \\
6.042 \\
-0.648 \\
0.394 \\
-9.869\end{array}$ & $\begin{array}{l}17.380 \\
6.679 \\
0.303 \\
0.037 \\
2.767\end{array}$ & $\begin{array}{c}211.059 \\
-7.077 \\
-1.243 \\
0.321 \\
-15.303\end{array}$ & $\begin{array}{c}279.337 \\
19.161 \\
-0.053 \\
0.467 \\
-4.434\end{array}$ & $\begin{array}{l}<0.001 \\
0.366 \\
0.033 \\
<0.001 \\
<0.001\end{array}$ \\
\hline
\end{tabular}


Table 2. (Continued)

\begin{tabular}{|c|c|c|c|c|c|c|}
\hline $\begin{array}{l}\text { Dependent } \\
\text { variables }\end{array}$ & $\begin{array}{l}\text { Independent } \\
\text { variables }\end{array}$ & $\begin{array}{c}\text { Linear regression } \\
\text { analysis } \beta \text {-coefficients }\end{array}$ & Standard error & $\begin{array}{l}95 \% \mathrm{Cl} \text {-lower } \\
\text { bound }\end{array}$ & $\begin{array}{l}95 \% \mathrm{Cl} \text {-higher } \\
\text { bound }\end{array}$ & P-value \\
\hline & SSB residuals (ml/d) & -0.093 & 0.019 & -0.130 & 0.056 & $<0.001$ \\
\hline & Energy 2002 (unit 40 kcal) & -0.022 & 0.233 & -0.480 & 0.435 & 0.923 \\
\hline & Energy residuals (unit 40 kcal) & 4.072 & 0.209 & 3.662 & 4.482 & $<0.001$ \\
\hline & & \multicolumn{5}{|c|}{$R^{2}=0.52, F(7554)=86.58, P<0.001$} \\
\hline \multirow[t]{9}{*}{ Sodium (mg) 2012} & Constant & 1453.912 & 349.153 & 768.085 & 2139.738 & $<0.001$ \\
\hline & Gender (male vs female) & -88.821 & 136.433 & -356.811 & 179.169 & 0.515 \\
\hline & Age 2002 & 2.656 & 6.143 & -9.409 & 14.722 & 0.666 \\
\hline & Sodium (mg) 2002 & 0.369 & 0.074 & 0.223 & 0.514 & $<0.001$ \\
\hline & Log SSB 2002 (ml/day) & 60.260 & 54.614 & -47.016 & 167.536 & 0.270 \\
\hline & SSB residuals (ml/day) & -0.208 & 0.383 & -0.961 & 0.545 & 0.588 \\
\hline & Energy 2002 (unit 40 kcal) & 6.644 & 4.925 & -3.029 & 16.318 & 0.178 \\
\hline & Energy residuals (unit $40 \mathrm{kcal}$ ) & 35.910 & 4.240 & 27.581 & 44.239 & $<0.001$ \\
\hline & & & $R^{2}=0.21, F(75$ & 4) $=21.28, P<0$ & 001 & \\
\hline \multirow[t]{9}{*}{ Ascorbic acid (mg) 2012} & Constant & 23.897 & 20.665 & -16.693 & 64.488 & 0.248 \\
\hline & Gender (male vs female) & -21.285 & 8.053 & -37.103 & -5.467 & 0.008 \\
\hline & Age 2002 & 0.778 & 0.365 & 0.061 & 1.495 & 0.033 \\
\hline & Ascorbic acid (mg) 2002 & 0.342 & 0.040 & 0.263 & 0.420 & $<0.001$ \\
\hline & Log SSB 2002 (ml/day) & 1.961 & 3.237 & -4.398 & 8.320 & 0.545 \\
\hline & SSB residuals (ml/day) & -0.013 & 0.023 & -0.058 & 0.032 & 0.569 \\
\hline & Energy 2002 (unit 40 kcal) & 0.415 & 0.226 & -0.029 & 0.858 & 0.067 \\
\hline & Energy residuals (unit $40 \mathrm{kcal}$ ) & 1.082 & 0.252 & 0.587 & 1.577 & $<0.001$ \\
\hline & & \multicolumn{5}{|c|}{$R^{2}=0.16, F(7554)=15.45, P<0.001$} \\
\hline \multirow[t]{9}{*}{ Cholecalciferol $(\mu \mathrm{g}) 2012$} & Constant & 0.877 & 0.342 & 0.205 & 1.549 & 0.011 \\
\hline & Gender (male vs female) & -0.045 & 0.133 & -0.307 & 0.216 & 0.733 \\
\hline & Age 2002 & 0.005 & 0.006 & -0.007 & 0.017 & 0.394 \\
\hline & Cholecalciferol ( $\mu \mathrm{g}) 2002$ & 0.139 & 0.035 & 0.071 & 0.207 & $<0.001$ \\
\hline & Log SSB 2002 (ml/day) & -0.058 & 0.054 & -0.164 & 0.047 & 0.279 \\
\hline & SSB residuals (ml/day) & -0.001 & 0.001 & -0.001 & 0.001 & 0.182 \\
\hline & Energy 2002 (unit 40 kcal) & 0.007 & 0.004 & 0.001 & 0.015 & 0.067 \\
\hline & Energy residuals (unit 40 kcal) & 0.028 & 0.004 & 0.020 & 0.037 & $<0.001$ \\
\hline & & \multicolumn{5}{|c|}{$R^{2}=0.12, F(7554)=10.73, P<0.001$} \\
\hline \multirow[t]{9}{*}{ Folic acid $(\mu \mathrm{g}) 2012$} & Constant & 0.446 & 0.544 & -0.623 & 1.515 & 0.413 \\
\hline & Gender (male vs female) & -0.142 & 0.213 & -0.559 & 0.276 & 0.506 \\
\hline & Age 2002 & 0.001 & 0.010 & -0.018 & 0.020 & 0.886 \\
\hline & Folic acid $(\mu \mathrm{g}) 2002$ & 0.069 & 0.010 & 0.050 & 0.089 & $<0.001$ \\
\hline & Log SSB 2002 (ml/day) & 0.090 & 0.086 & -0.078 & 0.258 & 0.295 \\
\hline & SSB residuals (ml/day) & 0.001 & 0.001 & -0.010 & 0.001 & 0.758 \\
\hline & Energy 2002 (unit 40 kcal) & -0.006 & 0.006 & -0.017 & 0.006 & 0.340 \\
\hline & Energy residuals (unit $40 \mathrm{kcal}$ ) & 0.001 & 0.007 & -0.012 & 0.015 & 0.829 \\
\hline & & \multicolumn{5}{|c|}{$R^{2}=0.08, F(7554)=7.289, P<0.001$} \\
\hline \multirow[t]{9}{*}{ Thiamine (mg) 2012} & Constant & 0.732 & 0.088 & 0.559 & 0.904 & $<0.001$ \\
\hline & Gender (male vs female) & 0.036 & 0.034 & -0.031 & 0.102 & 0.294 \\
\hline & Age 2002 & 0.004 & 0.002 & -0.003 & 0.003 & 0.977 \\
\hline & Thiamine 2002 & 0.230 & 0.035 & 0.162 & 0.298 & $<0.001$ \\
\hline & Log SSB 2002 (ml/day) & -0.020 & 0.014 & -0.047 & 0.007 & 0.141 \\
\hline & SSB residuals (ml/day) & 0.001 & 0.001 & 0.001 & 0.001 & 0.006 \\
\hline & Energy 2002 (unit 40 kcal) & 0.005 & 0.001 & 0.002 & 0.007 & $<0.001$ \\
\hline & Energy residuals (unit $40 \mathrm{kcal}$ ) & 0.018 & 0.001 & 0.016 & 0.020 & $<0.001$ \\
\hline & & \multicolumn{5}{|c|}{$R^{2}=0.45, F(7554)=63.466, P<0.001$} \\
\hline \multirow[t]{9}{*}{ Tocopherols (mg) 2012} & Constant & 4.611 & 1.750 & 1.174 & 8.048 & 0.009 \\
\hline & Gender (male vs female) & 0.556 & 0.680 & 1.892 & 0.781 & 0.414 \\
\hline & Age 2002 & 0.002 & 0.031 & -0.059 & 0.064 & 0.941 \\
\hline & Tocopherols (mg) 2002 & 0.394 & 0.042 & 0.311 & 0.477 & $<0.001$ \\
\hline & Log SSB 2002 (ml/day) & -0.127 & 0.274 & -0.665 & 0.410 & 0.642 \\
\hline & SSB residuals (ml/day) & 0.003 & 0.002 & -0.001 & 0.007 & 0.148 \\
\hline & Energy 2002 (unit 40 kcal) & 0.004 & 0.019 & -0.034 & 0.042 & 0.837 \\
\hline & Energy residuals (unit $40 \mathrm{kcal}$ ) & 0.135 & 0.020 & 0.090 & 0.180 & $<0.001$ \\
\hline & & \multicolumn{5}{|c|}{$R^{2}=0.20, F(7554)=19.78, P<0.001$} \\
\hline
\end{tabular}

Over the 10-year period, men's and women's BMI gained 0.3 and $0.7 \mathrm{~kg} / \mathrm{m}^{2}$ on average. Between 2002 and 2012, the mean energetic intake decreased from 2590 to $2421 \mathrm{kcal} /$ day for men and from 2026 to $1958 \mathrm{kcal} /$ day for women $(P<0.01)$. Mean sugar intake remained stable for men and for women, 13.5 to 13.1 energy-percent and 13.7 to 13.0 energy-percent, respectively. Mean daily intake of SSB decreased from 129 to $83 \mathrm{ml}$ in men and from 51 to $31 \mathrm{ml}$ in women. 
Table 2 presents the results of linear regressions with mineral and vitamin intake at 2012 as dependent variables, and baseline values for minerals, age, gender, energy intake, baseline log SSB, SSB residuals and energy residuals as independent variables. Calcium, iron and magnesium intake decreased with increasing baseline SSB intake, and with increasing SSB consumption during the 10 years. There was no relation between vitamins and SSB consumption, except for thiamine.

\section{DISCUSSION}

In this prospective study, only calcium, iron and magnesium decreased with increasing SSB consumption. This was not the case for other micronutrients, providing no evidence for a general micronutrient dilution. However, the decrease in calcium, iron and magnesium was associated with higher baseline SSB consumption and with increasing SSB consumption during the observed time period.

The relation between micronutrients and SSB can be influenced by the associated dietary pattern. For example, SSB consumption has been associated with lower daily consumption of fruits and vegetables, both micronutrient rich foods. ${ }^{1}$ This may indicate that a high SSB intake may be part of a general unhealthy behaviour, and that, when relating SSB to an outcome, it may be difficult to disentangle the specific health-related effect of SSB from other related unhealthy behaviours.

A major limitation of the present study is that of the original 1569 participants in 2002, 36\% returned for participation in 2012. However, the aim of our study was not to describe a representative sample of a population.
In conclusion, in this study, there was limited evidence of micronutrient dilution. Further research should be done to determine the clinical consequences on mineral intake.

\section{CONFLICT OF INTEREST}

The authors declare no conflict of interest.

\section{ACKNOWLEDGEMENTS}

We are indebted to the participants of this study. This research is accomplished by the Policy Research Centre Sport, which is funded by the Flemish government.

\section{AUTHOR CONTRIBUTIONS}

PC and $J L$ worked on the original idea for the study. PM analysed the data and drafted the first version of the manuscript. All the authors read and approved the final version of the review.

\section{REFERENCES}

1 Rennie KL, Livingstone MB. Associations between dietary added sugar intake and micronutrient intake: a systematic review. Br J Nutr 2007; 97: 832-841.

2 Gibson SA. Dietary sugars intake and micronutrient adequacy: a systematic review of the evidence. Nutr Res Rev 2007; 20: 121-131.

3 Livingstone MB, Rennie KL. Added sugars and micronutrient dilution. Obes Rev 2009; 10(Suppl 1): 34-40.

4 Devriese S, Huybrechts I, Moreau M, Van Oyen H. The Belgian Food Consumption Survey 1 - 2004. Scientific Institute of Public Health: Brussels, Belgium, 2006.

5 Duvigneaud $\mathrm{N}$, Wijndaele $\mathrm{K}$, Matton $\mathrm{L}$, Deriemaeker $\mathrm{P}$, Philippaerts $\mathrm{R}$ Lefevre $\mathrm{J}$ et al. Socio-economic and lifestyle factors associated with overweight in Flemish adult men and women. BMC Public Health 2007; 7: 23. 